\title{
Acute Airway Obstruction in a Uremic Patient After Undergoing Unsuccessful Bilateral Attempt of Permcath Catheterization
}

\author{
Ying-Ming Wang ${ }^{\mathrm{a}, \mathrm{b}}$, Hsiao-Hsien Ting ${ }^{\mathrm{a}}$, Yuan-Yi Chia ${ }^{\mathrm{a}}$, Kang Liu ${ }^{\mathrm{a}}$
}

\begin{abstract}
We report a case with a history of HIV infection and uremia who developed a prominent neck hematoma and sudden airway obstruction resulting in sudden cardiovascular collapse after failed bilateral attempted subclavian Permcath implantation for hemodialysis. The patient had a history of twice previous Permcath malfunction resolved by urokinase infusion. Postoperatively, venography revealed bilateral central vein stenosis. This report demonstrates that preoperative venography is important to assess the possibility of central vein stenosis for this type of patient. We also suppose that bilateral rather than unilateral neck hematoma is an important factor for the development of acute airway obstruction in this patient.
\end{abstract}

Keywords: Airway obstruction; Hematoma; Catheterization; Central venous

\section{Introduction}

Airway compression following neck hematoma has been reported as a complication of central venous cannulation. This is a life-threatening event that requires immediate and prompt intervention $[1,2]$. We report a uremic patient who suffered from acute airway obstruction followed by cardiac arrest inducing by a rapid developing neck hematoma after undergoing unsuccessful bilateral central venous catheterization.

\footnotetext{
Manuscript accepted for publication January 13, 2011

${ }^{a}$ Department of Anesthesiology, Kaohsiung Veterans General Hospital, and School of Medicine, National Yang-Ming University, Taiwan, R.O.C.

${ }^{\mathrm{b}}$ Corresponding author: Ying-Ming Wang, Department of Anesthesiology, Kaohsiung Veterans General Hospital, 386, Ta-Chung 1st Rd, Kaohsiung, Taiwan 813, R.O.C. Email: ymwang@vghks.gov.tw
}

doi:10.4021/jmc127w

\section{Case Report}

A 30-year-old mentally retarded man had a history of HIV infection that was complicated by end-stage renal disease under regular hemodialysis through Permcath dual catheter via left subclavian vein for five years. Two years ago, he had two instances of Permcath catheter obstructions resolved by urokinase infusion. On the current occasion, he was hospitalized due to pneumonia. After admission, Permcath malfunction was noted during his hemodialysis treatment, and femoral venous catheterization was performed for temporary hemodialysis. After antibiotic treatment, his pneumonia improved. Therefore, he was scheduled for Quinton Permcath implantation for future long-lasting hemodialysis.

Preoperative laboratory findings were within the normal range except for anemia with hemoglobin of $8.8 \mathrm{~g} / \mathrm{dl}$ and thrombocytopenia with platelet count of $94000 / \mu \mathrm{l}$. General anesthesia was induced with intravenous propofol $100 \mathrm{mg}$, fentanyl $100 \mu \mathrm{g}$, and rocuronium $50 \mathrm{mg}$. Laryngoscopy revealed a grade 1 view (Cormack and Lehane), and the patient's trachea was intubated using a $7.0 \mathrm{~mm}$ internal diameter cuffed oral endotracheal tube. Anesthesia was maintained with sevoflurane in oxygen/air. After use of full aseptic technique, an 18-gauge introducer needle was inserted into the left subclavian vein using real-time ultrasound guidance, and the J-wire was advanced into the vein. Initially, the guidewire was threaded smoothly and then difficulty was encountered when the guidewire was advanced beyond $12 \mathrm{~cm}$. The surgeon decided to advance the Pull-Apart Sheath/Dilator over the guidewire. However, the Pull-Apart Sheath could not be totally inserted over the guidewire. The surgeon subsequently tried to advance the Permcath catheter through the sheath, however, the difficulty was encountered when the catheter was advanced beyond about $12 \mathrm{~cm}$. Therefore, the procedure was failed. After short-term local compression of puncture site, the surgeon decided to perform right side subclavian Permcath catheterization. Unfortunately, the same problem occurred. The guidewire was advanced with difficulty at the length of $10 \mathrm{~cm}$ and the Pull-Apart Sheath could not be totally inserted over the guidewire. The surgeon gave up this procedure finally. After antagonism of neuromuscular block, 

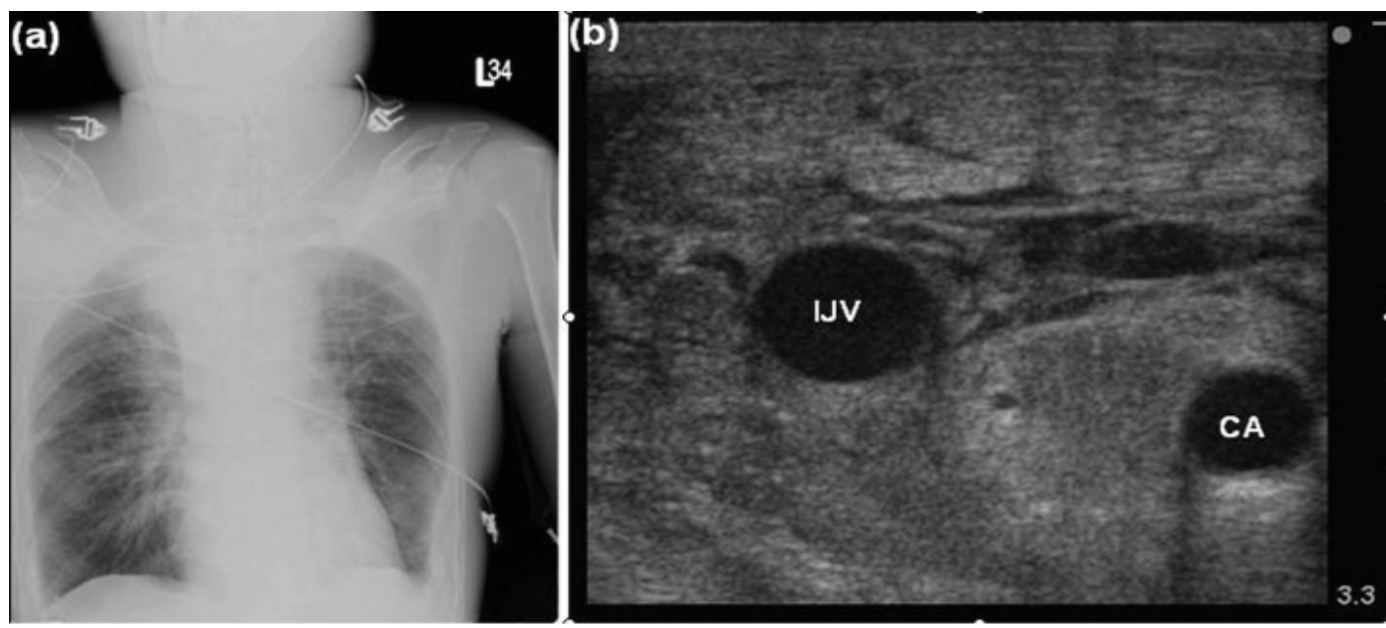

Figure 1. Evaluations post CPR. (a) The chest X-ray showed significant neck and upper chest soft tissue swelling, and upper mediastinal widening. (b) The sonography of the patient's neck revealed diffuse infiltration of hypoechogenic hematoma separating the common carotid artery (CA) and the internal jugular vein (IJV).

the patient breathed spontaneously and extubation was performed. Due to blood oozing from the puncture wound, bilateral local compressions were performed for 30 minutes. During this period, patient's consciousness returned. The patient's respiratory pattern was normal, and no apparent neck swelling was noted. The patient was then transferred to postanesthesia care unit (PACU).

However, five minutes later, respiratory distress developed gradually with mild paradoxical respiratory pattern, but oxygen saturation was $99 \%$ under a Venturi mask with $\mathrm{FiO}_{2}$ of $40 \%$. Bilateral breathing sounds were symmetric with slight basal rales without stridor. Hence we checked arterial blood gas (ABG) which showed: $\mathrm{pH} 7.167, \mathrm{PaCO}_{2}$ $64.7 \mathrm{mmHg}, \mathrm{PO}_{2} 94.5 \mathrm{mmHg}, \mathrm{HCO}_{3}-22.9 \mathrm{mmol} / \mathrm{L}, \mathrm{Hb} 8.7$ $\mathrm{g} / \mathrm{dl}$. Respiratory distress aggravated rapidly and significant neck swelling was noted. The patient began to exhibit agitation. In less than one minute, he lost consciousness, showing no chest wall movement. Assisted mask ventilation by ambu-bagging was applied. Soon, severe bradycardia occurred, followed rapidly by asystole. Cardiac massage and intubation were performed immediately and intravenous 1 mg epinephrine was given. Performing laryngoscopy during intubation was difficult (Cormack and Lehane grade 3) and it revealed significant supraglottic edema. The patient was resuscitated successfully within one minute. SBP returned to $120 \mathrm{mmHg}$ and heart rate was around 100 beats/min. ABG was analyzed again, revealing $\mathrm{pH} 7.076, \mathrm{PaCO}_{2} 58.9 \mathrm{mmHg}$, $\mathrm{PO}_{2} 312.6 \mathrm{mmHg}, \mathrm{HCO}_{3}-16.9 \mathrm{mmol} / \mathrm{L}, \mathrm{Hb} 7.7 \mathrm{~g} / \mathrm{dl}$. The patient regained consciousness quickly, and he then received intravenous midazolam $3 \mathrm{mg}$ for sedation to facilitate mechanical ventilation.

Prominent bilateral tense neck swelling with firm palpation was noted. Marked blood oozing from bilateral surgical wound was observed, and the surgeon compressed the wound for more than two hours to terminate bleeding. There was mild neck soft tissue swelling with no evidence of pneumothorax on the chest X-ray obtained immediately before CPR. The chest X-ray obtained post CPR revealed significant neck and upper chest soft tissue swelling and upper mediastinal widening (Fig. 1a). Subsequent investigation of the neck by ultrasound in PACU showed bilateral diffuse infiltration of hypoechogenic hematoma surrounding anterior neck (Fig. 1b). There was no evidence of puncture of subclavian artery or other arteries. Then, the patient was transferred to the intensive care unit. Unfortunately, he suddenly succumbed to refractory septic shock from uncertain infectious source on the postoperative fifteenth day.

\section{Discussion}

Many cases of cervical hematoma-induced acute airway obstruction following central venous catheter insertion have been documented. These obstructions are mainly caused by inadvertent arterial puncture-induced hematoma [1, 3], arterial pseudoaneurysm formation [4-6], and extravasation of intravenous fluid from the central catheter $[7,8]$. In this report, our patient suffered from acute respiratory compromise resulting from significant bilateral neck hematoma following bilateral subclavian venous puncture. The possible mechanisms of airway compression secondary to neck hematoma have been cited. Direct compression of the trachea by the hematoma can result in airway obstruction. Nevertheless, some authors disagreed and stated that the trachea was rigid and difficult to compress. They reported that pharyngolaryngeal edema secondary to venous and lymphatic obstruction by hematoma was the mechanism of airway obstruction [5, 9-11]. In our case, the vocal cords could not be visualized and pha- 
ryngeal tissues were edematous at laryngoscopy. In addition, retropharyngeal hematoma may be a possible mechanism for airway obstruction following subclavian vein and internal jugular vein cannulation $[2,12]$. Retropharyngeal space, a potential space lying between the middle (buccopharyngeal or alar fascia) and deep (prevertebral) layers of the deep cervical fascia, extends from the base of the skull to the level of T4 in the superior mediastinum and permits movement of the pharynx, esophagus, larynx, and trachea relative to the vertebral column during swallowing. Hemorrhage escaping from the neurovascular sheath via the needle puncture site into the retropharyngeal space and expanding in this large compartment can cause airway obstruction at different levels. As suggested, lateral neck X-ray could be a useful tool for diagnosis of retropharyngeal hematoma $[1,2,12]$. However, lateral neck X-ray was not performed in this case, so whether the retropharyngeal hematoma played a role in acute airway obstruction is unknown.

Airway dimension analysis in patients undergoing carotid endarterectomy by using preoperative and postoperative computed tomographic (CT) scan of the neck has been prospectively studied in a previous report [13]. Five of 19 patients with clinical evidence of airway obstruction were intubated postoperatively. Compared to non-intubated patients, the anterior-posterior and transverse diameter of the airway assessed by CT scan and the three-dimensional reconstructed airway volume were reduced to a greater extent in the intubated patients. Interestingly, the authors stated that neck swelling in the intubated group extended bilaterally from the operative side to the contralateral side of the neck. In the nonintubated patients, the neck swelling tended to be only unilateral. Significant bilateral neck swelling, resulting from the bilateral attempts at central venous catheterization, was noted in our patient, and acute airway obstruction developed subsequently even though there was no evidence of arterial puncture. This indicates that bilateral rather than unilateral neck swelling might have been an important factor for the development of acute airway obstruction in this patient.

Our patient was arranged venograpgy postoperatively which showed total occlusion of the bilateral brachiocephlic veins and superior vena cava. Central vein stenosis is commonly associated with central venous catheterization. The risk factors for central venous stenosis associated with central venous catheterization are multiple central venous catheter placements, longer catheter dwell times, subclavian vein catheterization (compared to internal jugular vein catheterization), left-sided catheterization, and catheter infections [14]. As central vein stenosis may be completely asymptomatic, and routine or serial venograms are not usually performed after central venous catheter placement or removal, the incidence of central venous stenosis is uncertain and likely to be underestimated. MacRae et al assessed the prevalence of central vein stenosis by using venography in 133 patients on chronic hemodialysis. Of this study popula- tion, $41 \%(55 / 133)$ had central vein stenosis. Of the 55 patients with central vein stenosis, 52 had a history of previous hemodialysis catheter insertion [15]. In one study evaluating subclavian vein stenosis before the placement of permanent vascular-access graft in dialysis patients, upper extremity venography showed $40 \%$ of the extremities with prior or existing subclavian catheters had significant stenosis. All 27 extremities without prior or existing subclavian catheters had normal venograms [16]. In addition, the patient had experienced several instances of Permcath malfunction that might have been a sign of central vein stenosis, even though the stenosis was asymptomatic preoperatively. Therefore, in patients with a history of previous multiple central venous catheterization and hemodialysis catheter malfunction, we suggest that preoperative venography is necessary for subsequent catheter insertions to assess the possibility of central vein stenosis or thrombosis and to avoid access failure and other complications.

Coagulopathy in our patient was associated with uremia and HIV infection. Though coagulopathy was not the main cause of neck hematoma in this case, we thought that it probably was an exacerbating factor in the rapid development of the neck hematoma in the absence of arterial puncture. Silva discovered only seven case reports of acute respiratory obstruction related to jugular vein cannulation in a Medline literature search of English-language publications since 1970, and five of these patients had coagulopathy [11].

Initial management of patients with acute airway obstruction secondary to neck hematoma should be directed at airway management. Generally, the treatment of choice for emergent airway obstruction is oral endotracheal intubation. Laryngoscopic intubation may be difficult due to an inability to visualize the normal supraglottic anatomy and epiglottis for anatomy distortion and pharyngolaryngeal edema. Failure to intubate where ventilation is not possible will result in the situation of "cannot ventilate, cannot intubate". In this critical situation, institution of a surgical airway by needle or surgical cricothyroidotomy, or by emergency tracheostomy, is indicated to prevent death $[7,17,18]$. However, in the presence of neck hematoma, it is important to be familiar with the procedures of cricothyroidotomy/emergency tracheostomy and to be aware that anatomical landmarks may be difficult to palpate. Laryngeal mask airway (LMA) has been reported to restore ventilation in acute airway obstruction caused by neck hematoma $[17,19,20]$. However, some limitations exist. The success of LMA rescue depends on the extent of airway edema. LMA could restore ventilation in the situation of airway obstruction caused by supraglottic edema, but LMA rescue would be unsuccessful in the presence of airway obstruction distal to the LMA such as edema of the glottis and tracheal edema [17]. Besides, alternative noninvasive approaches to difficult intubation such as fiberoptic intubation, use of intubating stylet or gum-elastic bougies, and blind oral/nasal intubation also have been reported in the 
management of airway obstruction caused by neck hematoma in previous reports $[11,19,21]$. In our case, fortunately, facemask ventilation was possible and tracheal intubation was successful during cardiac resuscitation. But, compared to oral intubation at anesthesia induction, tracheal intubation was more difficult because of significant supraglottic edema. Surgical evacuation of neck hematoma under local anesthesia is indicated when there is fatal airway obstruction or rapid expanding hematoma [22], but it can possibly fail to relieve the airway obstruction immediately because of potential laryngeal edema [9].

In summary, in this case, a bilateral neck hematoma developed because of bilateral venous puncture by a large bore cannula, vessel dilator, and catheter and grew rapidly because of compromised venous return through the stenotic central vein. Furthermore, the patient's bleeding tendencies impeded hemostasis and predisposed him to larger hematoma formation. Pharyngolaryngeal edema then developed and caused progressive airway obstruction. This report also illustrates that in patients with a history of previous hemodialysis catheter malfunction, preoperative venography is necessary before subsequent catheter insertions to assess the possibility of central vein stenosis or thrombosis and to avoid access failure and other complications.

\section{References}

1. O'Leary AM. Acute upper airway obstruction due to arterial puncture during percutaneous central venous cannulation of the subclavian vein. Anesthesiology 1990;73(4):780-782.

2. Lee M, Berger HW. Retropharyngeal hemorrhage and acute upper airway obstruction: a complication of catheterization of the internal jugular vein. Mt Sinai J Med 1981;48(1):56-57.

3. Larson E, Silberger M, Jakob K, Whittier S, Lai L, Della Latta P, Saiman L. Assessment of alternative hand hygiene regimens to improve skin health among neonatal intensive care unit nurses. Heart Lung 2000;29(2):136142.

4. Jeganathan R, Harkin DW, Lowry P, Lee B. Iatrogenic subclavian artery pseudoaneurysm causing airway compromise: treatment with percutaneous thrombin injection. J Vasc Surg 2004;40(2):371-374.

5. Kua JS, Tan IK. Airway obstruction following internal jugular vein cannulation. Anaesthesia 1997;52(8):776780 .

6. Cuhaci B, Khoury P, Chvala R. Transverse cervical artery pseudoaneurysm: a rare complication of internal jugular vein cannulation. Am J Nephrol 2000;20(6):476482.

7. Chakravarthy M, Thimmannagowda P, Jawali V. Life- threatening acute airway obstruction. J Cardiothorac Vasc Anesth 2005;19(5):659-660.

8. Clevens RA, Bradford CR. Airway obstruction secondary to central line intravenous fluid extravasation. Arch Otolaryngol Head Neck Surg 1994;120(4):437-439.

9. Munro FJ, Makin AP, Reid J. Airway problems after carotid endarterectomy. Br J Anaesth 1996;76(1):156-159.

10. Kwok OK, Sun KO, Ahchong AK, Chan CK. Airway obstruction following carotid endarterectomy. Anaesth Intensive Care 2004;32(6):818-820.

11. Silva FS. Neck haematoma and airway obstruction in a patient with goitre: complication of internal jugular vein cannulation. Acta Anaesthesiol Scand 2003;47(5):626629.

12. Stewart RW, Hardjasudarma M, Nall L, Mathews G, Davis R, 2nd. Fatal outcome of jugular vein cannulation. South Med J 1995;88(11):1159-1160.

13. Carmichael FJ, McGuire GP, Wong DT, Crofts S, Sharma S, Montanera W. Computed tomographic analysis of airway dimensions after carotid endarterectomy. Anesth Analg 1996;83(1):12-17.

14. Agarwal AK, Patel BM, Haddad NJ. Central vein stenosis: a nephrologist's perspective. Semin Dial 2007;20(1):53-62.

15. MacRae JM, Ahmed A, Johnson N, Levin A, Kiaii M. Central vein stenosis: a common problem in patients on hemodialysis. ASAIO J 2005;51(1):77-81.

16. Surratt RS, Picus D, Hicks ME, Darcy MD, Kleinhoffer M, Jendrisak M. The importance of preoperative evaluation of the subclavian vein in dialysis access planning. AJR Am J Roentgenol 1991;156(3):623-625.

17. Augoustides JG, Groff BE, Mann DG, Johansson JS. Difficult airway management after carotid endarterectomy: utility and limitations of the Laryngeal Mask Airway. J Clin Anesth 2007;19(3):218-221.

18. Digby S. Fatal respiratory obstruction following insertion of a central venous line. Anaesthesia 1994;49(11):10131014.

19. Garcia-Rodriguez CR, Yentis SM. Carotid artery puncture, airway obstruction and the laryngeal mask airway in a preeclamptic patient. Int J Obstet Anesth 1996;5(3):194-197.

20. Cook TM, Silsby J, Simpson TP. Airway rescue in acute upper airway obstruction using a ProSeal Laryngeal mask airway and an Aintree catheter: a review of the ProSeal Laryngeal mask airway in the management of the difficult airway. Anaesthesia 2005;60(11):11291136.

21. Jeganath V, McElwaine JG, Stewart P. Ruptured superior thyroid artery from central vein cannulation: treatment by coil embolization. Br J Anaesth 2001;87(2):302-305.

22. Senthuran S, Lim S, Gunning KE. Life-threatening airway obstruction caused by a retropharyngeal haematoma. Anaesthesia 1999;54(7):674-678. 